\title{
A REVIEW ON COMPARITIVE STUDY ON SAFETY AND EFFECTIVENESS OF IV FERRIC CARBOXY MALTOSE VERSUS IRON SUCROSE IN PATIENTS WITH IRON DEFICIENCY ANAEMIA OF CKD
}

\author{
LAYANA V..$^{1 *}$, RANI MANJU ${ }^{1}$, MATHEW GEORGE ${ }^{2}$, LINCY JOSEPH ${ }^{3}$ \\ ${ }^{1}$ Department of Pharmacy Practice, Pushpagiri College of Pharmacy, Thiruvalla- 689107, Kerala, \\ India. \\ ${ }^{2}$ Department of Pharmacology, Pushpagiri College of Pharmacy, Thiruvalla- 689107, Kerala, India. \\ ${ }^{3}$ Department of Pharmaceutical Chemistry, Pushpagiri College of Pharmacy, Thiruvalla- 689107, \\ Kerala, India.
}

\begin{abstract}
Deficiency of iron is one of the most common nutritional disorders in the society. Iron deficiency anemia is described as decreased production in red blood cells (RBCs) due to low body iron stores.Anemia commonly occurs in people with chronic kidney disease and it might begin to develop in the early stages and tends to worsen as disease progresses. Iron supplementation is mandatory in the majority of patients with renal disease, particularly in those receiving ESA therapy. Treatment with intravenous iron in some clinical situations could present some advantages over oral iron, such as faster and higher increases of hemoglobin $(\mathrm{Hb})$ levels and body iron stores. Some modern formulations of intravenous iron have emerged as a safe and effective alternative for iron deficiency anaemia management. E.g.: iron sucrose. Ferric carboxy maltose is a parenteral iron dextran-free product and the first of the new agents approved for rapid and high-dose replenishment of depleted iron stores.
\end{abstract}

Keywords: Iron deficiency anaemia, chronic kidney disease, hemoglobin, iron sucrose, ferric carboxy maltose

\section{INTRODUCTION}

Deficiency of iron is one of the most common nutritional disorders in the society. Iron deficiency anemia is described as decreased production in red blood cells (RBCs) due to low body iron stores. According to World Health Organization (WHO) Anemia is defined as a hemoglobin count below $13 \mathrm{~g} / \mathrm{dl}$ for adult males and post-menopausal women and below $12 \mathrm{~g} / \mathrm{dl}$ for premenopausal women. The main signs and symptoms of IDA are fatigue, breathlessness, weakness (Because in anemic patients the oxygen carrying capacity will be low, as a result it can lead to decreased oxygenation of skeletal muscle), pallor and rapid heartbeats ${ }^{(1)}$

Anemia commonly occurs in people with chronic kidney disease (CKD)-the permanent, partial loss of kidney function, it might begin to develop in the early stages of CKD and it tends to worsen as the disease progresses. Most people who have total loss of kidney function, or kidney failure, have anemia. ${ }^{(2)}$ IDA is common in chronic kidney disease (CKD) patients because of impaired intestinal absorption of dietary iron, blood loss, chronic inflammation, and increased iron requirements during therapy with erythropoiesis-stimulating agents (ESAs). A prevalent complication of CKD, IDA remains underdiagnosed and under treated. Both early-stage CKD and anemia can be asymptomatic, so renal and hematologic laboratory values should be monitored annually in at-risk patients. ${ }^{(3)}$

The kidney Disease Outcomes Quality Initiative (K/DOQI) concluded that serum ferritin and 
transferrin saturation (TSAT) levels should be the primary indicators for assessing iron management in patients with anemia and CKD. ${ }^{(4)}$ Iron supplementation is mandatory in the majority of patients with renal disease, particularly in those receiving ESA therapy. Intravenous and oral iron therapies are the main therapies for iron deficiency anaemia. The oral route is preferred to replace iron stores and mild to moderate iron deficiency anemia are treated using oral iron supplements. ${ }^{(1)}$ Treatment with oral iron supplements is simple, inexpensive and a relatively effective way of treating iron deficient conditions. If response doesn't occur at intervals 3-4 weeks of appropriate treatment, there is no reason to continue oral iron therapy.

Intravenous iron preparations have been used for treating iron-deficiency anaemia with a promising result and making it possible to avoid blood transfusion and side effects of oral iron preparation. The first iron intravenous preparations were associated with acute toxicity deriving from the release of free iron .Nowadays, all parenteral therapies are formulated so that each iron particle is surrounded by a carbohydrate molecule that permits a slow release of iron and limits toxicity. Current intravenous iron formulations include high or low molecular weight iron dextran, ferric gluconate, iron sucrose and, very recently, ferric carboxymaltose ${ }^{(5)}$. They all share the same structure, but differ from each other by the size of the core and the identity and density of the surrounding carbohydrate. Iron sucrose has been widely used for treating anaemia with promising result. The efficacy and safety of IS have been established. Multiple dosing is required for IS which decreases the compliance.

Ferric carboxymaltose (FCM) is non-dextran containing intravenous iron agent, having a very low immunogenic potential and so not predisposed to high risk of hypersensitivity reactions, designed to be administered in large doses in a short period of time, with very less side effects overcoming the limitations of the existing intravenous iron agents. The safety and efficacy of FCM in patients with iron-deficiency with significant improvement of $\mathrm{Hb}$ level have been reported previously ${ }^{(6)}$. FCM is cost effective and requires less frequent hospital visits which improve patient compliance.

\section{REVIEW OF LITERATURE}

1) Suyajna D. Joshi, et $a \boldsymbol{l}^{(7)}(2016)$ conducted Comparative study of efficacy and safety of intravenous ferric carboxy maltose versus iron sucrose in treatment of postpartum iron deficiency. Women less than 10 days after delivery with hemoglobin between $\geq 6 \mathrm{~g} / \mathrm{dl}$ and $\leq 11 \mathrm{gm} / \mathrm{dl}$ requiring iron supplementation were enrolled. 200 women of postpartum iron deficiency anemia were allocated into two groups. Iron sucrose group, subjects were given I.V. iron sucrose in multiple doses, $200 \mathrm{mg} /$ day on day $0,2,4,6,8$ total of $1000 \mathrm{mg}$. FCM group ,subjects were given I.V. ferric carboxymaltose $1000 \mathrm{mg}$ single dose. Both teams haemoglobin\%, and serum ferritin were done on 0 and day 30 of last dose of parenteral iron. Side effects, compliance were noted. There was statistically significant rise of $\mathrm{Hb}$ in FCM group compare to iron sucrose group. Mean rise of serum ferritin was significantly higher in ferric carboxy maltose group compared to iron sucrose. No serious adverse events were reported in either the FCM group or Iron sucrose group. The study concludes that Properties like ultra-short duration of treatment, fewer adverse reactions and better compliance makes FCM the first-line drug in the management of postpartum iron deficiency anemia.

2) Prof. Dr. SangeetaShah et al $\mathbf{l}^{(8)}(2018)$ conducted a Comparative Study Of Efficacy And Safety Of Intravenous Ferric Carboxymaltose Versus Intravenous Iron Sucrose In The Treatment Of Iron Deficiency Anaemia of Pregnancy. This was a Comparative Observational Prospective Study done over a period of one year. 60 antenatal women were studied. Iron sucrose was given in a dose of 200 $\mathrm{mg}$ intravenously in $200 \mathrm{ml}$ normal saline over a period of $15-20$ min on alternate days until the required total dose was administered; not 
exceeding the maximum dose of 1000 $\mathrm{mg} /$ week. Patients were observed for side effects or anaphylactic reactions. Any minor or major side effects were documented. Hemoglobin test was repeated at the end of 4 weeks interval. In this study mean percentage change in hemoglobin levels post treatment in group IS was less compared to group FCM. Side effects were noted in $36.7 \%$ of women in group IS and $33.3 \%$ of women in group FCM. Based on the results of this study it may be concluded that, the intravenous iron carboxymaltose is more effective in the treatment of iron deficiency anaemia among pregnant women compared to intravenous iron sucrose. Further it is well tolerated in pregnant women as side effects are comparable to that of iron sucrose.

3) Muzaffer Keklik et $\boldsymbol{a l}^{(9)}$ (2017), conducted a study to Evaluate Iron Sucrose and Ferric Carboxymaltose Therapies in Patients with Iron Deficiency Anemia. The aim of this retrospective study was to assess the efficacy of intravenous ferric carboxymaltose (FCM) and iron sucrose (IS) treatments in patients with IDA.In this retrospective study, overall data obtained from 180 patients undergoing FCM and IS treatments . In all patients, the following laboratory evaluations were performed before treatment began: complete blood count $(C B C)$, serum iron level, total iron-binding capacity (TIBC), TS, and serum ferritin level. An expected increase in hemoglobin $(\mathrm{Hb})$ and transferrin saturation (TS) levels was observed in the two groups. Also ferritin increase was greater in the FCM group, compared to IS group. Results indicate that when compared with IS administered in five infusions of $200 \mathrm{mg}$ each, FCM administered in one or two infusions of 500-1000 mg given 1 week apart is a safe and effective alternative for the treatment of IDA. Moreover, FCM appears to provide a better and more rapid correction of serum ferritin levels in patients with IDA than IS.

4) Nalini Sharma et $\boldsymbol{a l}^{(10)}(2017)$ conducted Comparative Study of Efficacy and Safety of Ferric Carboxymaltose Versus Iron Sucrose in Post-partum Anaemia. In this prospective, comparative study, 120 post-partum women with iron-deficiency anaemia ( $\mathrm{Hb} \backslash 10 \mathrm{~g} \%)$ were divided into two groups. A fixed dose of 1000 $\mathrm{mg}$ of FCM or IS was given within ten days of delivery. $\mathrm{Hb}$ and blood serum ferritin were repeated fourteen days post transfusion. There is a mean increase in $\mathrm{Hb}$ and ferritin in both the groups. This study concludes that, FCM was very effective in improving $\mathrm{Hb}$ concentration as well as in early replenishment of iron stores in patients with PPA. Large doses given in a short period of time not solely save hospital resources however additionally improve patient satisfaction. It has important benefit to be used within the outpatient department. From this study, we can recommend its use in post-partum women with iron-deficiency anaemia.

5) S.Vikrant et $\boldsymbol{a l}^{(11)}(2015)$ conducted a study on safety and efficacy of high dose ferric carboxy maltose in patients with chronic kidney disease. It was a prospective observational study and ferric carboxy maltose was administered as iv infusion. Efficacy was evaluated by comparing $\mathrm{Hb} /$ serum iron status at the first follow up visit. A total of five hundred infusions were administered to 450 patients. It concludes that increase in $\mathrm{Hb}$ was observed uniformly across all stages of chronic kidney disease and high dose administration of ferric carboxy maltose was safe and well tolerated.

6) Antonio Robalo Nunes et $a l^{(12)}(2017)$ conducted a study on Efficacy and Tolerability of Intravenous Ferric Carboxymaltose in Patients with Iron Deficiency. The aim was to evaluate the efficacy and tolerability of FCM treatment in patients with iron deficiency, with or without anemia, at a hospital outpatient clinic. Data was collected retrospectively from medical records. During this 2-year study, 459 patients were included. Mean age was $58.6 \pm$ 17.5 years and most patients received cumulative FCM doses of 501-1000mg (63.2\%). Six weeks after administration of FCM, efficacy endpoints hemoglobin increase $\geq 2 \mathrm{~g} / \mathrm{dL}$, hemoglobin increase $\geq 3 \mathrm{~g} / \mathrm{dL}$, and transferrin saturation $>20 \%$ were attained by $41 \%, 20 \%$, and $63 \%$ of patients, respectively. Treatmentemergent adverse events were documented in $<4 \%$ of patients. In conclusion, FCM treatment 
was effective and well-tolerated by outpatients with iron deficiency at a hospital clinic, and its dosage should be adjusted to improve iron deficiency management in clinical practice.

\section{CONCLUSION}

This study showed FCM was very effective in improving $\mathrm{Hb}$ concentration as well as in early replenishment of iron stores. Due to properties like ultra-short duration of treatment

i.e. ability to administer $1000 \mathrm{mg}$ doses in a single sitting, fewer adverse reactions and better compliance makes FCM the first-line drug. Large doses given in an exceedingly short period of time not solely save hospital resources however additionally improve patient satisfaction. It has important benefit to be used within the outpatient department. And also, among the IV preparations Ferric carboxy maltose has a greater safety profile and offers faster elevation of haemoglobin and iron stores as compared to iron sucrose.

\section{REFERENCE}

1. Naveen Kumar Panicker, Hridya, Raghuveer Prabhu; Comparison of Efficacy and Safety Profile of Oral Iron Formulations in Patients with Iron Deficiency Anemia; International Journal of Pharmaceutical Sciences Review and Research ;2016 Dec;41(2):248-252

2. Brugnara $\mathrm{C}$, Eckardt $\mathrm{KU}$; Hematologic aspects of kidney disease. In: Taal MW, ed. Brenner and Rector's The Kidney. 9th ed. Philadelphia: Saunders; 2011: 2081-2120.

3. Krikorian S, Shafai G, Shamim K; Managing iron deficiency anemia of CKD with IV iron. US Pharmacist.;2013;38(8):22-26.

4. Wish JB; Assessing iron status: beyond serum ferritin and transferrin saturation; Clin J Am Soc Nephrol. 2006:S4-S8.

5. Auerbach $M$, Ballard $H$; Clinical use of intravenous iron: administration, efficacy, and safety; Hematol Am Soc Hematol Educ Program.;2010;2010:338-47.

6. Klaire E, Nancy T, Andrea A, Atif K, Shahed A; Efficacy and safety profile of single dose
IV FCM in the management of renal anaemia-a single centre experience. Nephrol Dial Transplant;2013;28(1):363-4.

7. Suyajna D. Joshi, Shaila Chikkagowdra, Veerendra Kumar CM; Comparative study of efficacy and safety of intravenous ferric carboxy maltose versus iron sucrose in treatment of postpartum iron deficiency anemia; International Journal of Reproduction, Contraception, Obstetrics and Gynecology; 2016 Aug;5(8):2566-2570

8. Prof. Dr. SangeetaShah, Dr. K. Swapna ;A Comparative Study Of Efficacy And Safety Of Intravenous Ferric Carboxymaltose Versus Intravenous Iron Sucrose In The Treatment Of Iron Deficiency Anaemia Of Pregnancy; IOSR Journal of Dental and Medical Sciences; 2018 September;17(9): 13-17.

9. Muzaffer Keklik, Uğur Kalan, Serdal Korkmaz, Gülşah Akyol, Bilal Aygün, Ertuğrul Keklik; Evaluation of Iron Sucrose and Ferric Carboxymaltose Therapies in Patients with Iron Deficiency Anemia; Erciyes Med J 2017; 39(2): 59-62.

10. Nalini Sharma, J .Lalnunnem Thiek,Tanie Natung,Santa Singh Ahanthem; Comparative Study of Efficacy and Safety of Ferric Carboxymaltose Versus Iron Sucrose in Post-partum Anaemia; The Journal of Obstetrics and Gynecology of ;2017August ; 67(4):253-257.

11. S. Vikrant and A. Prashar; Safety and efficacy of high dose ferric carboxy maltose in patients with chronic kidney diseas; Indian Journal of Nephrology; 2015 ;25(4);213-221

12. António Robalo Nunes,Ana Palricas Costa,Sara Lemos Rocha, and Ana Garcia de Oliveira; Efficacy and Tolerability of Intravenous Ferric Carboxymaltose in Patients with Iron Deficiency at a Hospital Outpatient Clinic:A Retrospective Cohort Study of Real-World Clinical Practice; Hindawi ;2017; Article ID 3106890:7 pages 